\title{
WORLD'S FIRST APPLICATION OF NEW RAIL HARDENING TECHNOLOGY AND STATE-OF-THE-ART WATER- COOLING PROCESSES FOR LONG ROLLING*
}

\author{
Emanuele Osto ${ }^{1}$ \\ Federico Pegorin ${ }^{2}$ \\ Luigi Ettore Giacomini
}

\begin{abstract}
In late 2013, the installation of the world's first application of a break-through technology for rail hardening was started at Baogang Iron \& Steel (Group) Co. Ltd. in Inner Mongolia, China, one of world's leading rail producers. The "Injector Dualphase Rail Hardening" (idRHa+) system was developed by Primetals Technologies and Centro Sviluppo Materiali S.p.A. (RINA-CSM) a Technical Center headquartered in Rome, Italy. Baogang was a key partner, providing its large expertise of premiumrail producer, including its experience with existing offline technologies. With a design based on thermal, mechanical and metallurgical models, idRHa+ can apply customized cooling protocols, so to obtain the desired microstructure and hardness distribution across the rail head, with a high degree of accurateness and operation flexibility. idRHa+ can be installed in inline or offline configurations. During 2014, the system was successfully commissioned at rail mill \#2 in the city of Baotou, Inner Mongolia, and entered the regular commercial production. This paper gives an overview of the key concepts behind idRHa+'s design, the progression of its commissioning and testing till the ramp-up to consistent industrial operation. Derived from idRHa+ research and experience, other water-cooling technologies are available for different applications such as: quenching \& tempering of medium-large sections, control of distorsion of merchant bars, quenching of stainless grades for improving the resistance to corrosion.
\end{abstract}

Keywords: Head-hardening; Controlled cooling; Quenching; Carbide precipitation. 


\section{INTRODUCTION}

Heavy-haul and high-speed railways call for long rails with precise linear tolerances and increased resistance to wear and rolling contact fatigue. This drives the development of the new rail grades as well as of improved manufacturing processes. The "Injector Dual-phase Rail Hardening" (idRHa+) system was jointly developed by Primetals Technologies and Centro Sviluppo Materiali S.p.A. (RINA-CSM). In late 2013, the installation of the world's first application of a break-through technology for rail hardening was started at Baogang Iron \& Steel (Group) Co. Ltd., one of the world's leading rail producers, whose expertise greatly contributed to the project success.

idRHa+ provides tailored inline cooling protocols which serve to obtain the accurate fine-pearlitic microstructure and hardness distribution across the rail head. It can process with a unique level of flexibility more than 150 tph of rails in a wide range of sizes and grades according to the updated requirements of the main international rail Standards.

The design of idRHa+ is based on thermal, mechanical and metallurgical models, validated through experimental trials in a full-scale industrial pilot plant for rails.

\subsection{Rail Market Scenario and Trends}

In 2013 , the yearly production of rails in the world has reached a stable volume over 12 million tons, sustained by demographic, economic, political and technological drivers. While in the mid-term the overall production capacity is expected to quickly approach the mark of 15 millions tons per year, there are strong indications that a further growth will lead to 20 millions tons per year by the 2020's decade.

A network density close or higher than $0.05 \mathrm{~km} / \mathrm{km}^{2}$ is currently installed only in some limited areas of the world (figure 1), but represents a possible medium-term target for many other areas in most of the continents. Beside such network extension, there is a tendency towards the installation of better quality tracks, mainly in connection with the developments of high-speed or/and heavy-haul transport lines.

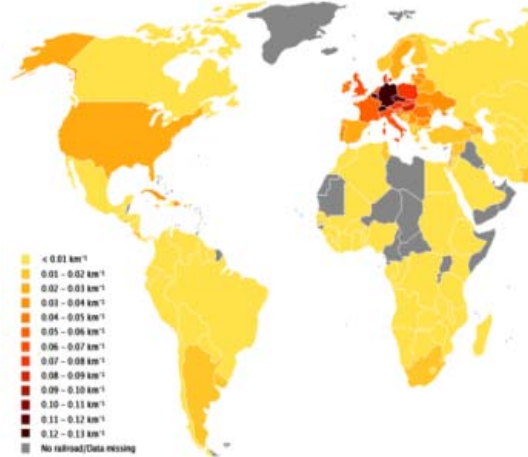

Figure 1: Rail network density

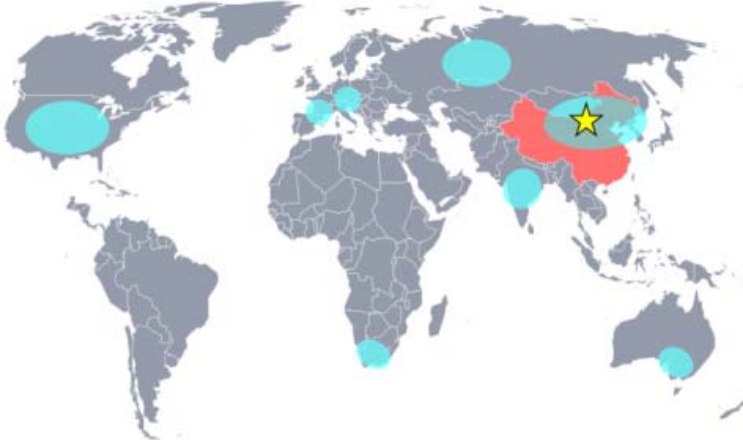

Figure 2: Baotou (China) and other rail production hubs in the world

The technical and the quality requirements of the rails are indicated in the relevant country (or region) standards. The quick impulse of development of the rail technical qualities and performances, has therefore determined a corresponding need to update many Standards. Clear examples of this trend are the new EN 13674-1:201104 (Europe), ASTM A1-2010 (USA), GOST 51685-2013 (Russia), the Indian IRS T12-2009 and Chinese TB/T 2344-2012 (China). 
It is notable that in the years 2010-2013 the Standards of the countries with significant hubs of rails production (figure 2) were updated, a clear indication of the dynamism of the technological evolution in this field. The new Standards classify the requirements for the rails in every aspects, and gradually introduce new premium-rail categories. Increased as-rolled length, tighter size tolerances and improved resistance to rolling contact fatigue and wear: these requirements set the new global trends (figure 3 ).

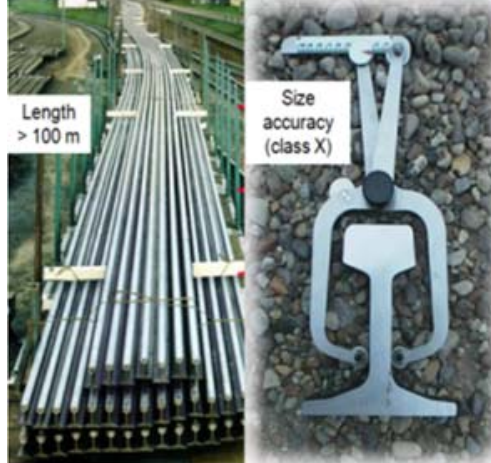

Figure 3. Requirements of premium-rails processes
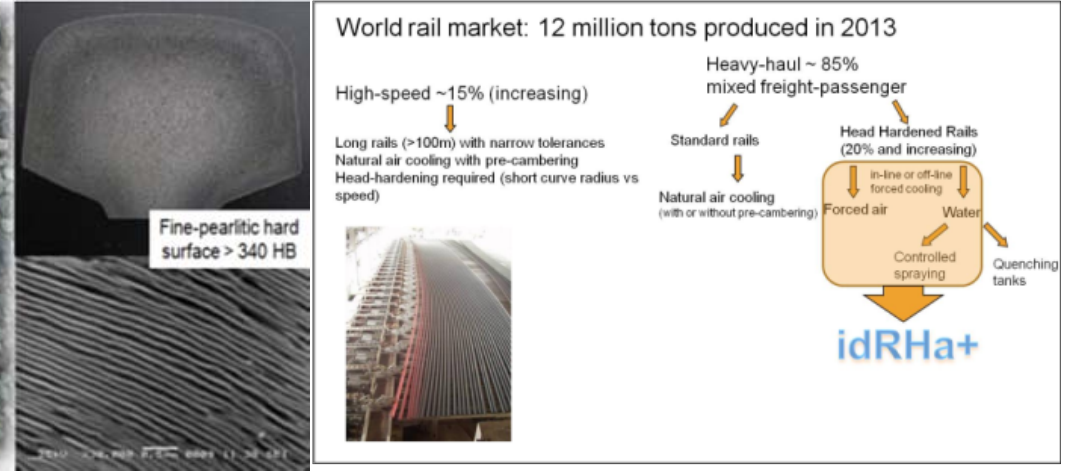

Figure 4. Rail market with technological

High-speeds railways are increasingly employed for passenger rail transportation, as they are competitive and practical against air transportation over short to medium distances. Cruising speeds exceeding $350 \mathrm{~km} / \mathrm{h}$ are not uncommon, and in 2014 high-speed lines currently shared $15 \%$ of the market, with a trend to increase. On the other hand, freight transportation calls for large load capability. Long freight trains with more than one hundred cars (the "mile train") are frequently used for commodities like minerals and crops, while the individual axle load increased from the conventional 25 tons to heavy-haul 40-50 tons. Mixed freight-passengers railways currently represent $85 \%$ of the market. This fast progression of rail technical and economical requirements challenges the rail manufacturers to become flexible and reactive in the world market, as they must evolve their practices and processes through incremental innovations. Addressing this scenario, major process technologies are nowadays available (figure 4), to enable the efficient production of a wide range of grades-sizes-lengths of rails, with quick automatic setup operations and self-adaptability of use. Integrated modeling techniques interface with intelligent process control systems, so that all the process areas may be effectively governed and the conversion costs minimized.

\section{BACKGROUND OF BAOGANG'S PROJECT}

Baogang is the largest rail manufacturer in the world, with an overall rail production capacity up to 1.36 million tons (record reached in 2009). In 2011, Baogang had noticed that the Chinese market of hot rolled common rails was on a downturn, while heat-processed rails were instead increasingly requested by the railway builders. Also on the rise was the international market for rails characterized by high hardness. Baogang soon realized that, in order to answer these new calls from the rail market, it would be imperative to install an innovative inline hardening facility to produce hardened and high-strength rails for both the domestic and international markets. Consequently, Baogang undertook a broad-scale study to examine the advantages and disadvantages of the available air and atomized-mist cooling solutions. The investigation led Baogang to the decision to cooperate with Primetals Technologies, which was awarded a contract for the first idRHa+ system in December 2012. 
Baogang decided to equip its rail rolling mill \#2 in Baotou, Inner Mongolia, with a new idRHa+ thermal process for heavy-duty rails (figure 5 ). This mill has a yearly capacity of 400,000 metric tons of rails, produced in several sizes and grades, for both domestic and export markets.

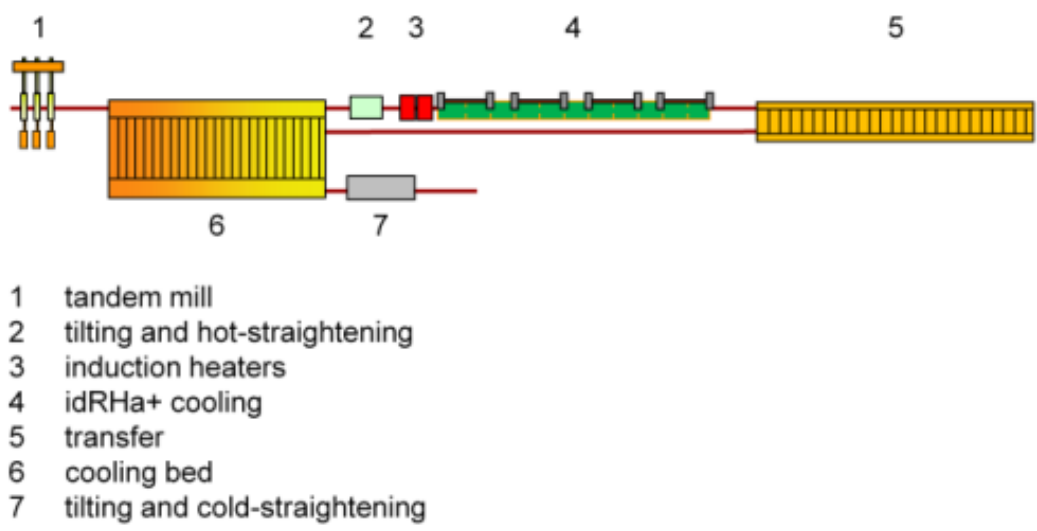

Figure 5. Sketch layout of idRHa+ line at Baogang's mill

The project consisted in realizing a state-of-the-art heat treatment process of the rail head, through the development of an industrial air and mist cooling technology. The main goals were to enhance the rail production chain, to be quickly applicable in a brownfield situation like the mill in Baotou with the smallest possible impact, and to extend the product varieties to satisfy customers' requirements in the world.

The execution of the project proved that idRHa+ is a very flexible process for air and mist cooling, easy to control, with a high cooling capacity and suitable to heat-treat all different kinds of rail grades and types. All the project's expectactions were satisfied, and a stable product quality obtained in compliance to the requirements of the applicable technical standards.

The idRHa+ process and technology was designed and developed by Primetals Technologies and Centro Sviluppo Materiali S.p.A. (RINA-CSM). RINA-CSM, headquartered in Rome, Italy, is a private and market-oriented industrial technical center, which focuses on research and development of materials and relevant processes. Baogang was a key partner, providing its large expertise of premium-rail producer, including its experience with existing offline technologies. ABP Induction Systems, headquartered in Dortmund NRW, Germany, was the qualified supplier of the induction heating technology. Since the very beginning of the project and down through its installation and commissioning, efforts were profusely dedicated to it by all the stakeholders according to a working model of close cooperation (figure 6).

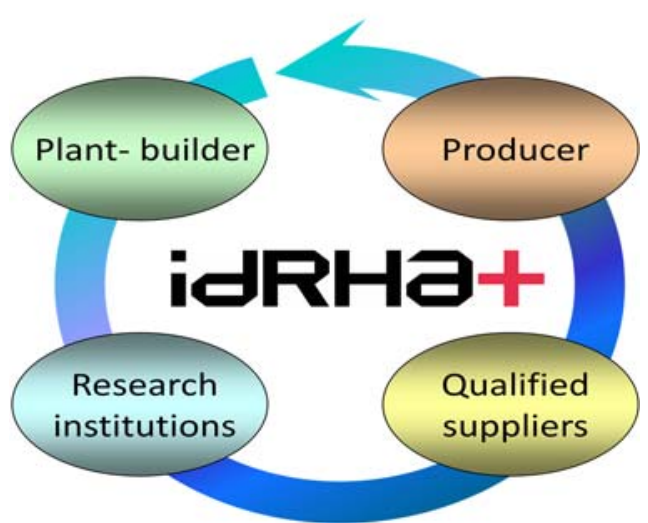

Figure 6. Cooperation among the project stakeholders

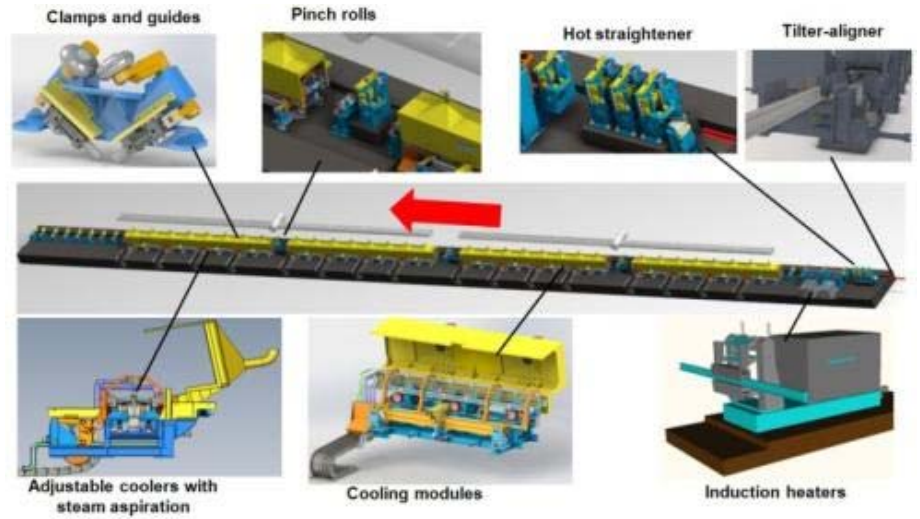

Figure 7. Main components of idRHa+ system 


\section{1 idRHa+ line and Equipment}

The idRHa+ head-hardening line comprises a group of integrated devices (figure 7), each with a specific functional and technological purpose.

The idRHa+ line is positioned after the rolling finishing stand (figure 8).
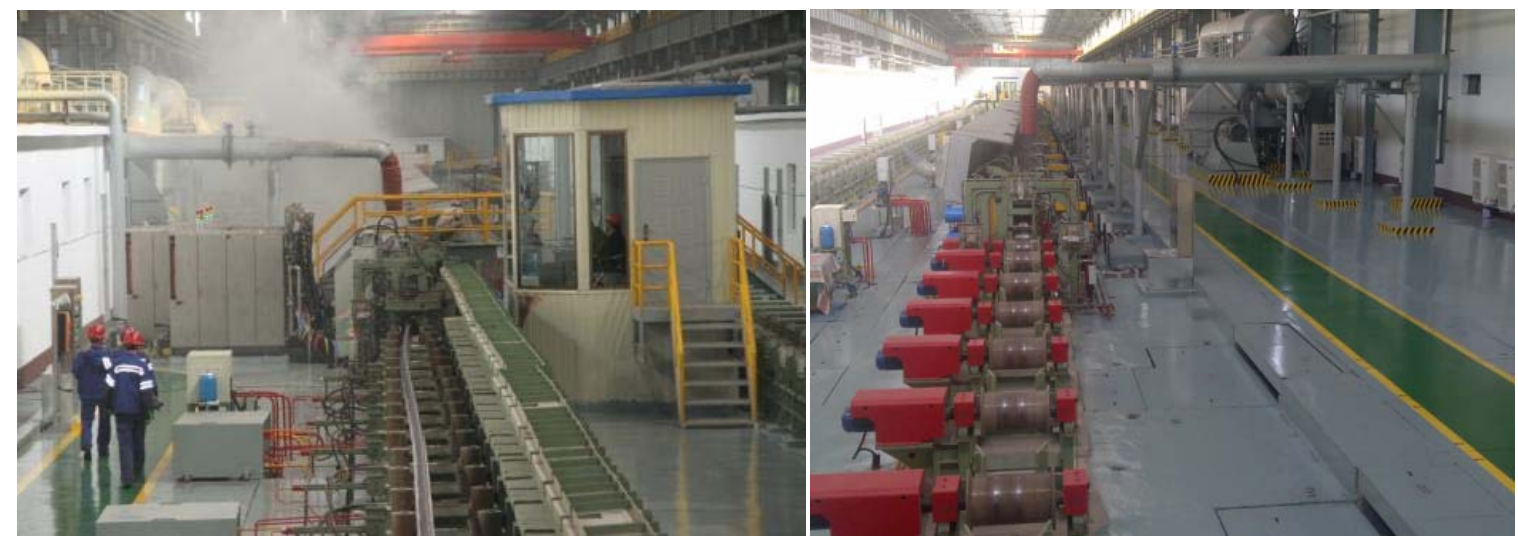

Figure 8. Views of idRHa+ line at Baogang's mill

The rail is lifted in vertical position by a tilter whose center of rotation can be laterally moved and adjusted to every size of rail.

The hot rolled rail could present some undesirable bends or waved parts along its length, especially in the first meters at the two ends. Therefore, before headhardening, a hot-straightening unit with vertically adjustable driven rolls applies a soft plastic deformation to the rail so that a suitable straightness can be recovered. This preliminary operation ensures that the rail can be smoothly processed without ramming into any component.

After preliminary straightening, induction heaters with high-energy density provide the thermal energy to equalize, whenever necessary, the temperature along the rail length and obtain a suitable temperature distribution across the rail section. To obtain the desired temperature gradients in selected areas of the rail (head and foot), an adequate heating power is provided. The parameters of the induction units are preliminarly calculated by a FEM modeling analysis and then dynamically tuned during the operation, according to the actually measured evolution of the temperature profile.

The induction heaters are specially shaped coils fed by individual power converters, which are designed according to Insulated Gate Bipolar Transistor (IGBT) technology (figure 9).

The coils are top-bottom split for the head and the foot of the rail, which grants the maximum efficiency and control with an optimized consumption. The heaters are adjustable along both the vertical and the horizontal axes, in order to fit the residual out-of-straightness of the rail and to accomodate the different sizes and shapes (figure 10). The rail is kept guided through the inductors by horizontal driven and vertical rolls while the risk of contact of the rail against the ceramic walls is prevented by means of a contour-follower device with rollers. 
Individual powered coils

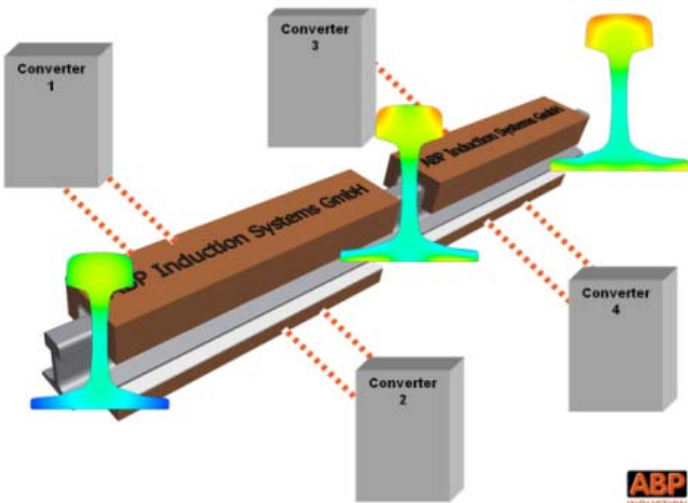

Figure 9. Induction coil feeding

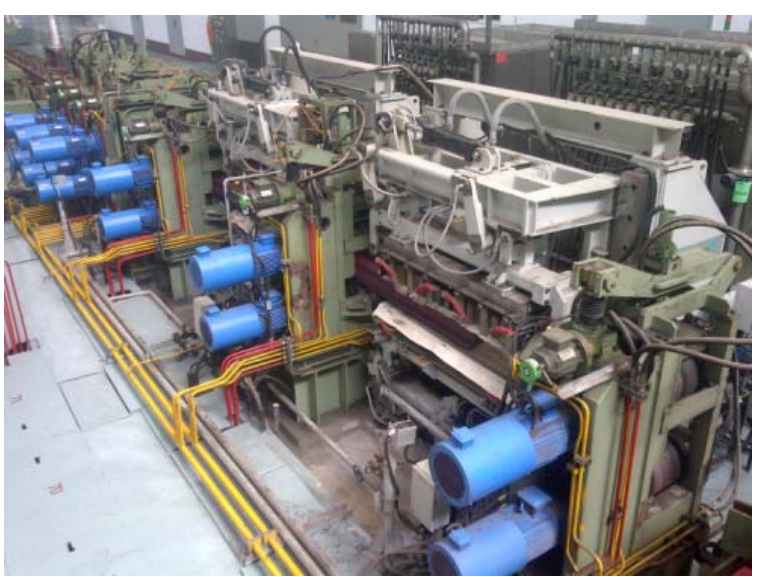

Figure 10. Induction heaters by ABP Induction Sys

Immediately after leaving the induction heaters, the rail enters the cooling area, which is the core of the idRHa+ technology. It is composed by several modules grouped in sections. Each module is equipped with a set of coolers mounted on metal frames. The total length of the line grants a sufficient time to obtain the desired percentage of transformed austenite in the rail head, that is more than $50 \%$ on the head surface and more than $20 \%$ in the head core.

The coolers are mist-atomizing nozzles which are adjustable at different mix of media. The nozzles selectively act on a part of the rail in order to grant the heat transfer coefficient required to reach the desired result. The coolers have also the function to keep the temperature gradients in the rail section balanced, so that uncontrolled rail deformations are avoided. Air-jet blades are also provided in the modules as necessary. The proper combination of the coolers along the line and their setting flexibility guarantee that an optimal processing strategy may be applied and maintained. The position of the coolers is adjustable by means of a cam-system, to maintain the proper distance from the rail which is necessary for an optimized cooling effect. The position adjustment also permits to fit the system to the different rail sizes and geometries.

The frames with the coolers and the sealing covers of each module may be hydraulically swung open, providing an easy access during maintenance (figure 11).

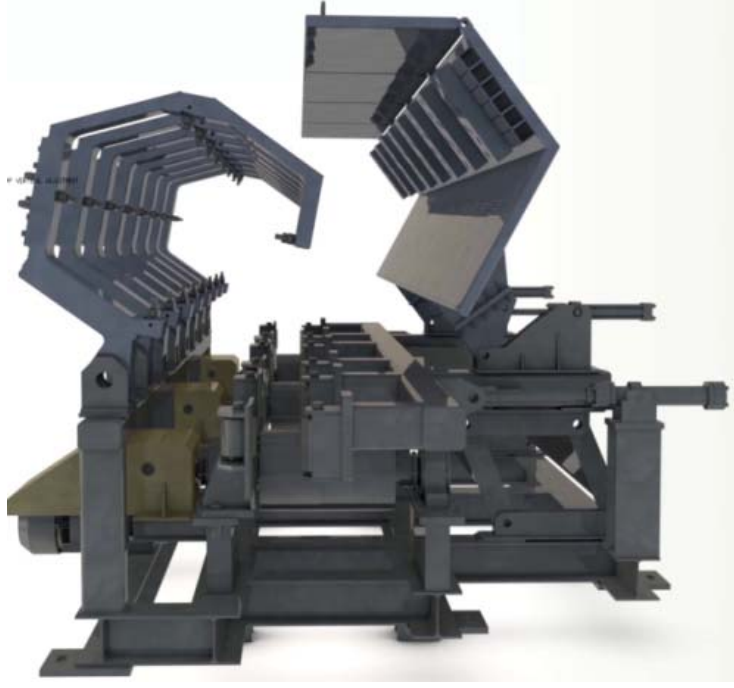

Figure 11 Front view of idRHa+ module

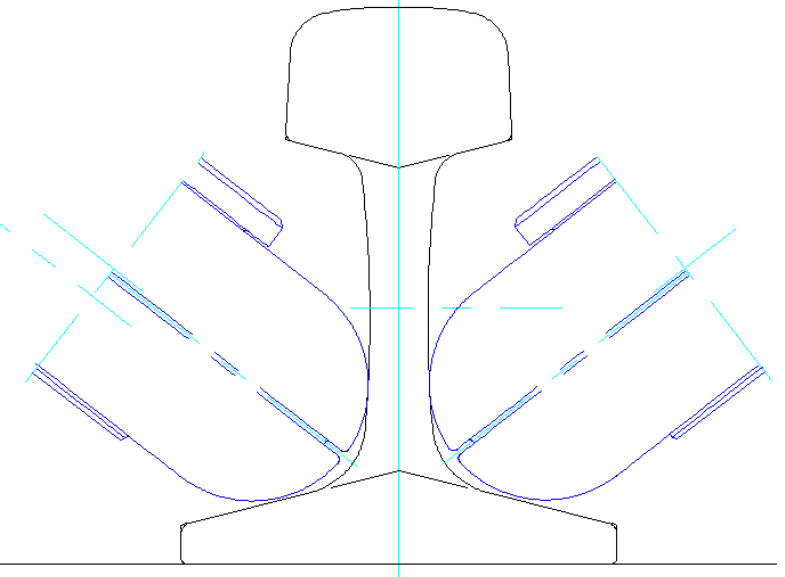

Figure 12 Rail guiding rollers

Each module is fitted with a suction system to remove the steam. Inside the modules, hydraulically swinging rollers keep the rail secured on its web and guided while moving through the coolers (figure 12). Between two subsequent modules, horizontal 
pinch-rolls with vertical idle rollers hold the rail centered and guided to prevent any distortion.

After the idRHa+ line, the treated rail reaches the cooling bed and is further cooled to the room temperature by natural air cooling.

\section{2 idRHa+ Process}

At idRHa+ entrance, the rail surface entry temperature is kept in the range of $800 \div 900^{\circ} \mathrm{C}$ by using the residual rolling heat, integrated by the selective induction heating. The cooling rate is typically adjustable in the range of $0.5 \div 20^{\circ} \mathrm{C} / \mathrm{s}$ as function of desired microstructure and final mechanical characteristics. The rail leaves idRHa+ with a temperature in the range of $350 \div 450^{\circ} \mathrm{C}$ depending on the rail grade. The process strategy (e.g. heating rate, cooling rate, temperature profile) is pre-defined as a function of the final product properties. The process control system, a platform named PROMOD, uses several embedded thermal, mechanical and metallurgical models. The cooling strategy calculated by PROMOD is applied and fine-tuned during the hot commissioning of the line, by taking into account the actual measurement of parameters and their prediction during the rail process route.

This guarantees the application of an ideal cooling path all along the rail length and through each position in the rail cross section. Deviations of the cooling path are strictly monitored and controlled, so that areas with too high or too low hardness, as well as any undesired microstructure, can be avoided. Temperature set points at each section of the line are monitored by thermoscan pyrometers, whose readings are connected to the process control (figure 13).

Figure 13 Single-line process control model

Figure 14 Basic principles of controlled cooling strategy

Corrective cooling actions are applied in order for the requested cooling strategy to be precisely and effectively implemented. Erratic or out-of-tolerance readings set off appropriate alarms (figure 14).

A specific temperature drop in function of time is proposed in order to have the austenite transformed at the exit of idRHa+ in a percentage higher than $50 \%$ on rail surface and $20 \%$ at rail head core. Figure 15 shows a typical cooling path obtainable with idRHa+ for pearlitic rail, and figure 16 the corresponding model of austenite transformation behavior. 


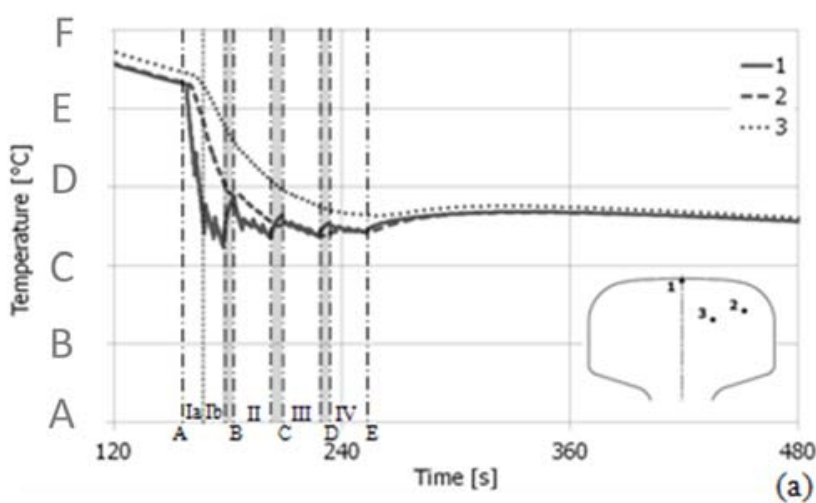

(a) Figure 15 Typical cooling path with idRHa+

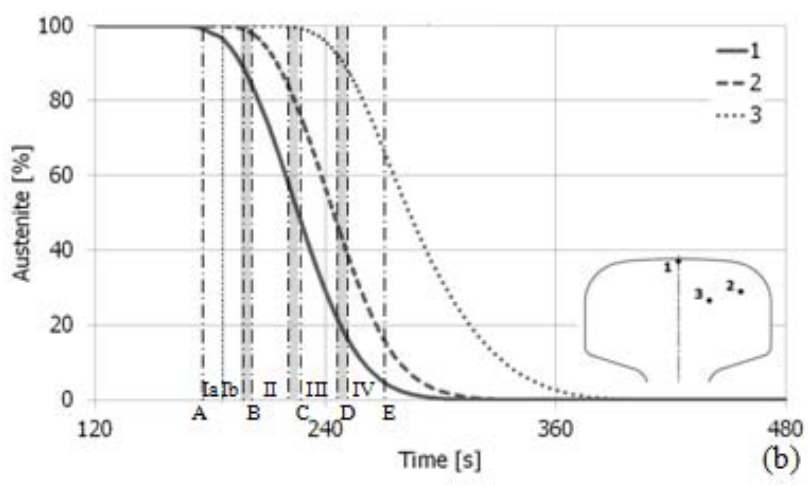

Figure 16 Model of austenite transformation

\section{3 idRHa+ Testing in Pilot Plant}

The numerical models of idRHa+ must be tested and validated through an extensive set of experimental trials, which are done in the pilot installation at RINA-CSM laboratory. The pilot plant is equipped with all the necessary regulation devices and controls to simulate any combination of settings and of parameters (figure 17). Specifically for Baogang's project, several rail materials were tested, including U75V, $\mathrm{U} 71 \mathrm{Mn}$ and U76CrRe. This allowed for a large variety of cooling strategies to be validated. During each test, all parameters were recorded and cross-checked with the predicted values from the numerical models.

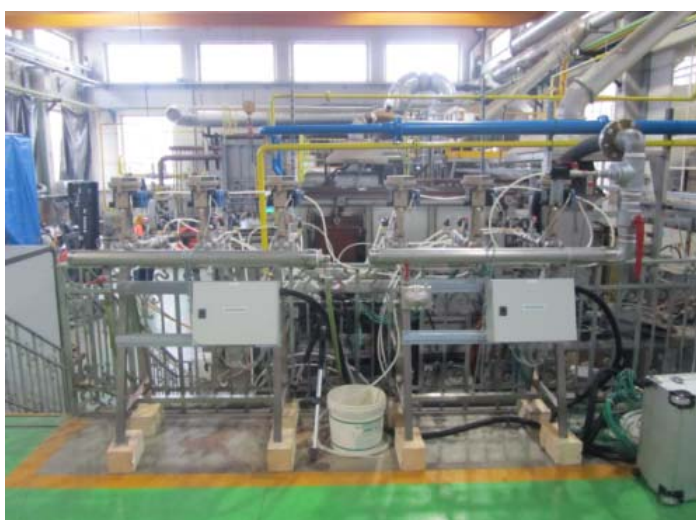

Figure 17 Pilot plant at RINA-CSM laboratory

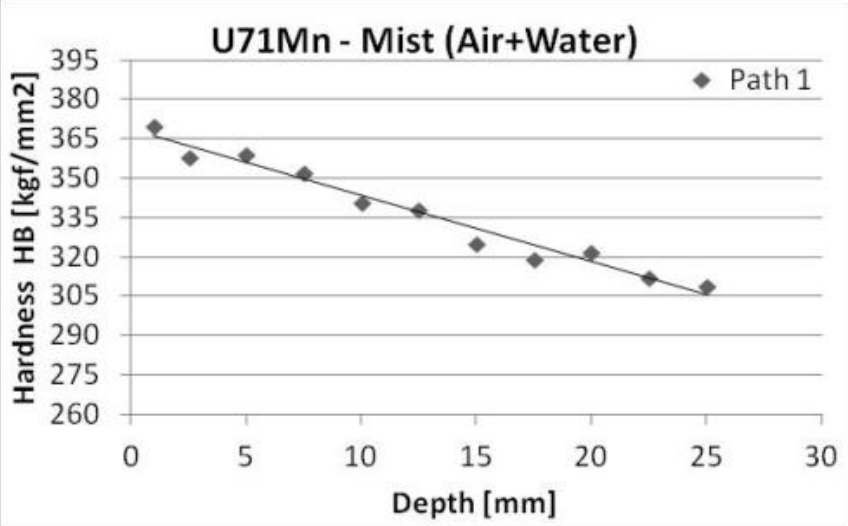

Figure 18 Hardness profile on rail head

The main measured parameters are:

- $\quad$ tensile strength;

- $\quad$ hardness profile on rail head (figure 18);

- $\quad$ hardness across the rail section;

- $\quad \mathrm{KIC}$ fracture toughness;

- microstructure investigation. 
The close collaboration with qualified manufacturers offered the opportunity to develop special components with higher performances and precisely fit for idRHa+. Several types of components were tested to determine the ideal design choices. In particular, the behavior and process features of different types of mist-atomizing nozzles and jet-air blades were deeply studied, to determine their best mounting configuration (figure 19).

Figure 19 Pilot plant with mist-atomizing nozzles

Figure 20 Rail sample equipped with several thermo-couples

Several samples of different qualities of rails were equipped with thermo-couples (up to 9 on a rail section) to record with high accuracy the cooling paths across the rail section (figure 20). The cooling paths were then used to tune the numerical models and, superimposed to the CCT curves of the material, to determine and track the ideal metallurgical transformation path.

The metallurgical microstructures and the technical features of the rail were investigated to verify the capability of the system to meet the requirements. This served to identify the ideal cooling strategy to be implemented in the industrial application. This scientific approach allowed to identify and control many of the factors which influence the process, like chemistry, pressure and flow parameters of the used cooling media, nozzles types, etc. An example cooling strategy is indicated in figure 21 , and the resulting metallurgical structure and hardness profile are shown in figure 22. 


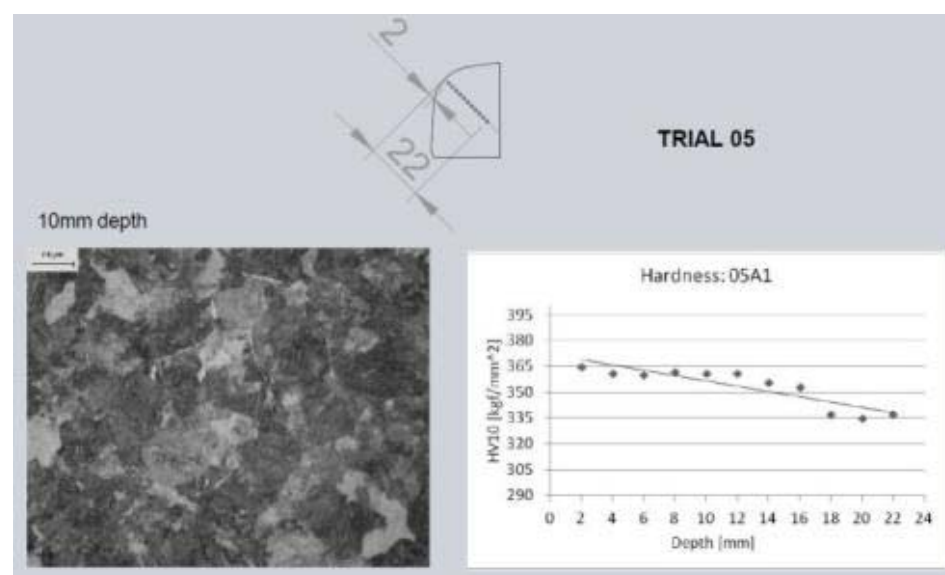

Figure 22 Example of metallurgical structure and hardness profile

\subsection{Commissioning of idRHa+ at Baogang}

Only sixteen months after Baogang had awarded the contract for idRHa+, the commissioning phase of the project commenced on April 19, 2014. Already in August 2014, a small quantity of rails had been successfully hardened at Baosteel mill.

By the end of October 2014, already 23,000 tons of hardened rails had been produced in several grades, including H320 $50 \mathrm{~kg} / \mathrm{m}, \mathrm{H} 34050 \mathrm{~kg} / \mathrm{m}, \mathrm{H} 34060 \mathrm{~kg} / \mathrm{m}$ and H350 UIC54.

The idRHa+ line can now process up to 18 pieces per hour, with an average of 15 pieces per hour with a $100 \mathrm{~m}$ standard length.

The relentless efforts by the experts and engineers from the involved parties were decisive to the fast commissioning and start-up phases, which were completed through a steadily advancing optimization of the equipment and process (figure 23).

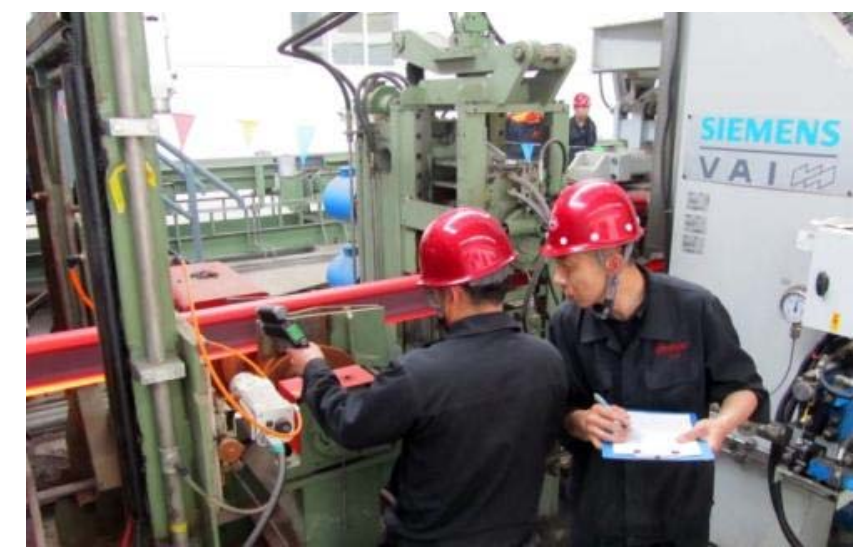

Figure 23 Temperature measurements during commissioning

\subsection{Tests on U75V}

A large amount of rails (approximately 300 ) was tested during the commissioning, implementing systematic and consistent methods and protocols (figure 24). 


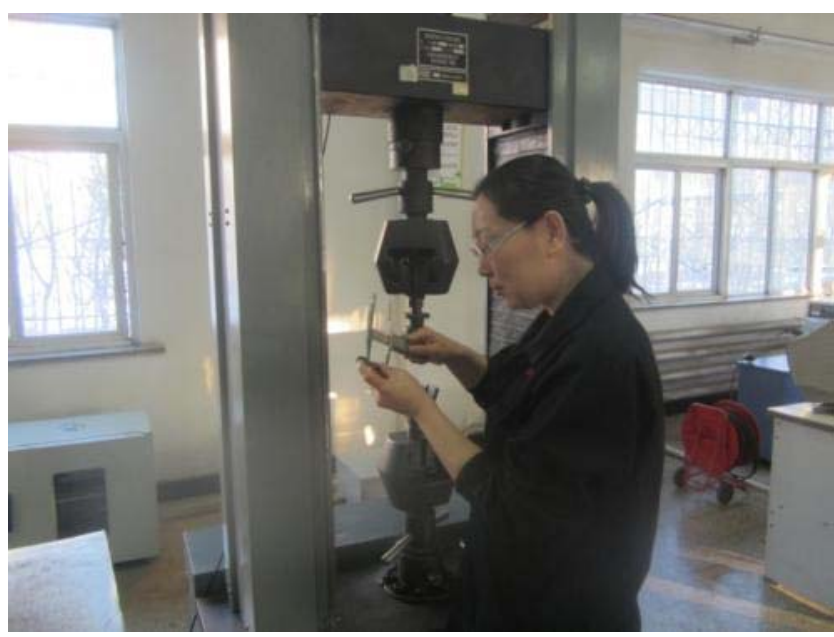

Figure 24 Systematic testing at rail mill in Baotou

For each U75V $60 \mathrm{~kg} / \mathrm{m}$ processed rail, five samples were examined (figure 25), and the following parameters investigated on both the head surface and across the head section: hardness, tensile strength and microstructure. The test results confirmed the stability and consistency of idRHa+ process.

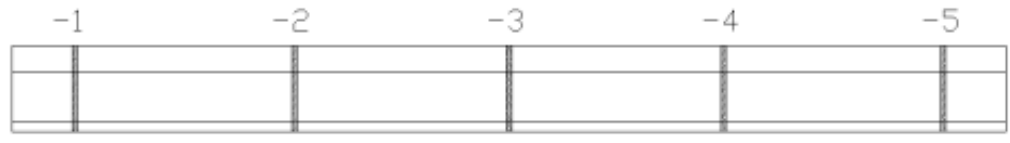

Figure 25 Rail samples for tests

\subsection{Hardness On the Rail Head Surface}

The hardness on the rail head surface was tested on a HB3000 Brinell machine per conditions HBW 10/3000/15. Five points were tested on each sample, and measurements read with BRINtronic-MD automatic microscope. The results are indicated in table 1.

Table 1 Hardness values on rail head surface

\begin{tabular}{|c|c|c|c|c|c|c|c|}
\hline Position & 1 & 2 & 3 & 4 & 5 & Max. $\Delta$ & Total $\Delta$ \\
\hline-1 & 374 & 367 & 378 & 371 & 365 & 13 & \multirow{5}{*}{24} \\
\hline-2 & 365 & 358 & 364 & 359 & 354 & 11 & \\
\hline-3 & 359 & 361 & 365 & 360 & 359 & 6 & \\
\hline-4 & 368 & 366 & 364 & 360 & 360 & 8 & \\
\hline-5 & 360 & 368 & 368 & 367 & 358 & 10 & \\
\hline Standard & \multicolumn{5}{|c|}{$\geq 340 \mathrm{HB}$} & $\leq 30 \mathrm{HB}$ & $\leq 30 \mathrm{HB}$ \\
\hline
\end{tabular}

\subsection{Hardness Across the Rail Head Section}

The hardness across the rail head section was tested on an EDMJ20 Rockwell machine. With idRHa+ mist-atomizing process, all the measured data were within the standards prescribed for U75V (figure 26). 


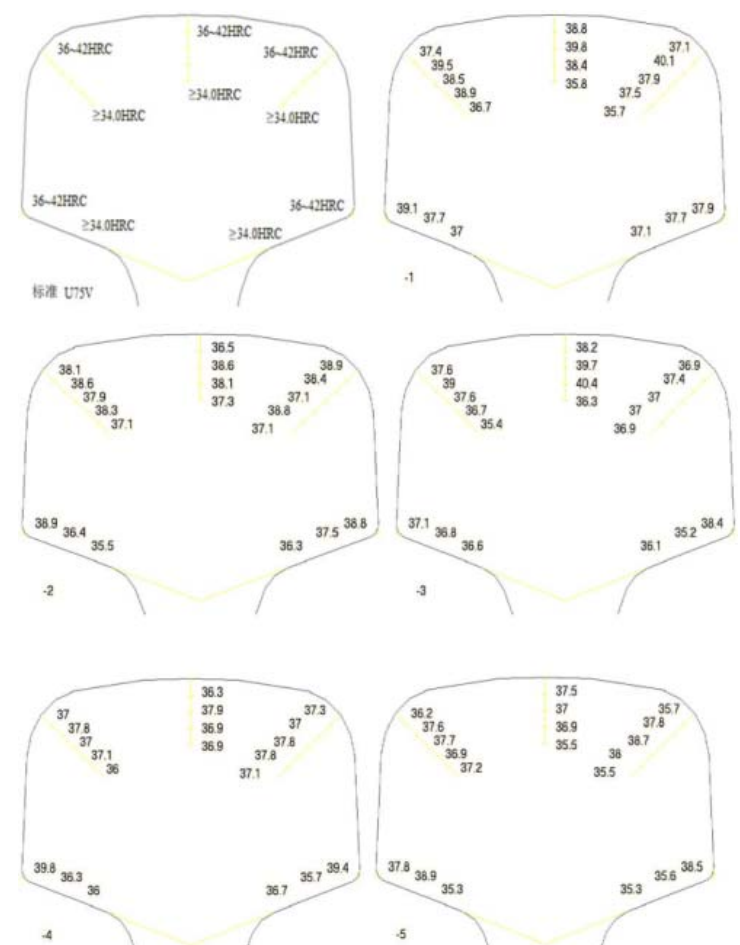

Figure 26 Hardness values across the rail head section

The depth of the hardened layer across the rail head is one of the most important targets of the rail heat treatment process. Tests showed the hardness reaching the $37.4 \mathrm{HRC}$ value at $30 \mathrm{~mm}$ from the surface, and falling below $34 \mathrm{HRC}$ only at positions deeper than $45 \mathrm{~mm}$ from the surface, as indicated by figure 27 , which also includes a 500x micrograph showing the fine perlite structure obtained in the depth range of 5 to $25 \mathrm{~mm}$ from the surface.

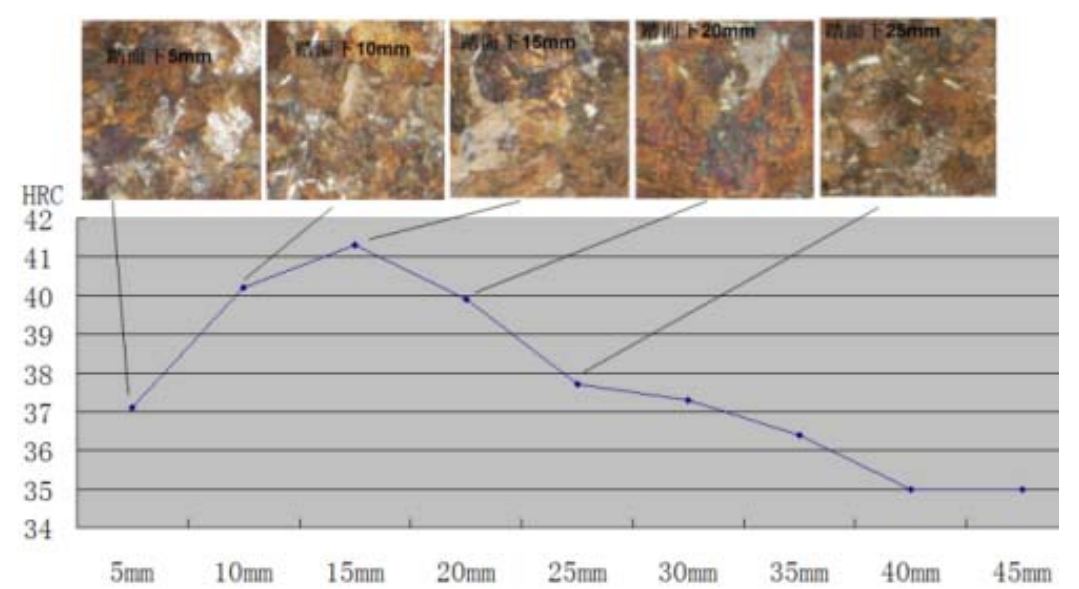

Figure 27 Depth of hardened layer and micrographs across the rail head

\subsection{Tensile strength and elongation}

The tensile strength and elongation were tested on two specimens on each sample. The two specimens had a $10 \mathrm{~mm}$ diameter, and were symmetrically taken from both sides of the rail head. The results are indicated in table 2 .

\subsection{Microstructure}

Microstructure investigation showed the predominance of fine pearlite with a little quantity of, as indicated in table 2. 
Table 2. Test results of ultimate tensile strength (Mpa) and elongation (\%)

\begin{tabular}{|c|c|c|c|c|c|}
\hline \multirow{2}{*}{$\begin{array}{c}\text { Position } \\
\text { Standard }\end{array}$} & \multicolumn{2}{|c|}{} & \multicolumn{2}{|c|}{ M } & \multirow{2}{*}{ Microstructure } \\
\cline { 2 - 5 } & $\geq 1180$ & $\geq 10$ & $\geq 1180$ & $\geq 10$ & \\
\hline-1 & 1201 & 10.5 & 1227 & 11 & Fine Pearlite + few ferrite \\
\hline-2 & 1225 & 10 & 1214 & 13 & Fine Pearlite + few ferrite \\
\hline-3 & 1211 & 11 & 1210 & 11 & Fine Pearlite + few ferrite \\
\hline-4 & 1217 & 11 & 1210 & 10 & Fine Pearlite + few ferrite \\
\hline-5 & 1185 & 12 & 1206 & 11.5 & Fine Pearlite + few ferrite \\
\hline
\end{tabular}

\subsection{Stability of idRHa+ Process}

To verify the stability and operational reliability of idRHa+ process, the tests were repeated on a large amount of rails (approximately 300 ). idRHa+ confirmed to be a very stable process, with results and performances presenting no significant difference with respect to the full air process already in place and accepted by the market (Table 3).

Table 3. Summary of recorded measurements during commissioning

\begin{tabular}{|c|c|c|c|c|c|c|}
\hline $\begin{array}{l}\text { Cooling } \\
\text { method }\end{array}$ & Sample & $\begin{array}{c}\text { Tensile } \\
\mathrm{MPa}\end{array}$ & Elongation & $\begin{array}{c}\text { Surface } \\
\text { Hardness } \\
\text { HB }\end{array}$ & $\begin{array}{l}\text { Min. value on } \\
1^{\text {st }} \text { point in } \\
\text { section } \\
\text { HRC }\end{array}$ & $\begin{array}{c}\text { Min. value of } \\
\text { inner section } \\
\text { HRC }\end{array}$ \\
\hline Air & \multirow{2}{*}{$\begin{array}{l}\text { Min. } \sim \text { Max. } \\
\text { Average/sample } \\
\text { numbers }\end{array}$} & $\frac{1182 \sim 1286}{1247 / 27}$ & $\frac{10 \sim 14}{12.4 / 27}$ & $\frac{343 \sim 380}{359 / 27}$ & $\frac{36.5 \sim 39}{37.5 / 27}$ & $\frac{36.0 \sim 39.0}{37.1 / 27}$ \\
\hline Mist & & $\frac{1186 \sim 1275}{1216 / 78}$ & $\frac{10 \sim 13.5}{11.3 / 78}$ & $\frac{348 \sim 365}{357.7 / 39}$ & $\frac{36 \sim 40.8}{37.8 / 65}$ & $\frac{34.3 \sim 40.8}{37.2 / 65}$ \\
\hline \multicolumn{2}{|r|}{ Standard } & $\geq 1180$ & $\geq 10$ & $340 \sim 400$ & $36 \sim 42$ & $\geq 34$ \\
\hline
\end{tabular}

\subsection{Milestones of idRHa+ at Baogang}

Effective and professional teamwork among the project partners resulted in a very compact schedule, with less than two years from the early concept definition to the commencement of the commercial production, according to the main milestones:

- 2012 February - preliminary discussions in Baotou;

- 2012 May to July - pilot plant trials, simulating the proposed cooling process;

- 2012 December - contract award;

- 2013 - design, procurement, construction, installation;

- 2014 April - commencement of hot commissioning;

- 2014 September - performance certificate for U75V R60 rail processed by idRHa+;

- 2014 October - commercial production of U75V R60 gr. 340 with 15 pcs/h @100 m length.

\section{CONCLUSION}

In order to answer the demanding requirements from the premium-rail market, Baogang decided to install an innovative inline hardening facility in its rail mill \#2, located in Baotou, to produce hardened and high-strength rails for both the domestic and international markets. idRHa+ process and technology represent the cuttingedge hardening solution, which enables a controlled microstructure and hardness distribution to be obtained across the rail head. The idRHa+ project execution model effectively integrated the skills and expertises from Baogang, Primetals Technologies, CSM and ABP, which resulted in a very compact overall schedule. The world first application of idRHa+ technology commenced the regular commercial production at Baogang's mill in October 2014. 